Supporting Information

\title{
Tailoring the Electron-Rich Moiety in Benzothiadiazole-Based Polymers for Efficient Photocatalytic Hydrogen Evolution Reaction
}

\author{
Giane B. Damas ${ }^{1}$, Cleber F. N. Marchiori ${ }^{2}$, C. Moyses Araujo ${ }^{1}$ \\ ${ }^{1}$ Materials Theory Division, Department of Physics and Astronomy, Uppsala University, Box \\ 516, 75120 Uppsala, Sweden. \\ ${ }^{2}$ Department of Chemistry - Angström Laboratory, Uppsala University, Box 538, 75121 \\ Uppsala, Sweden.
}

\section{1-Optimized geometries}

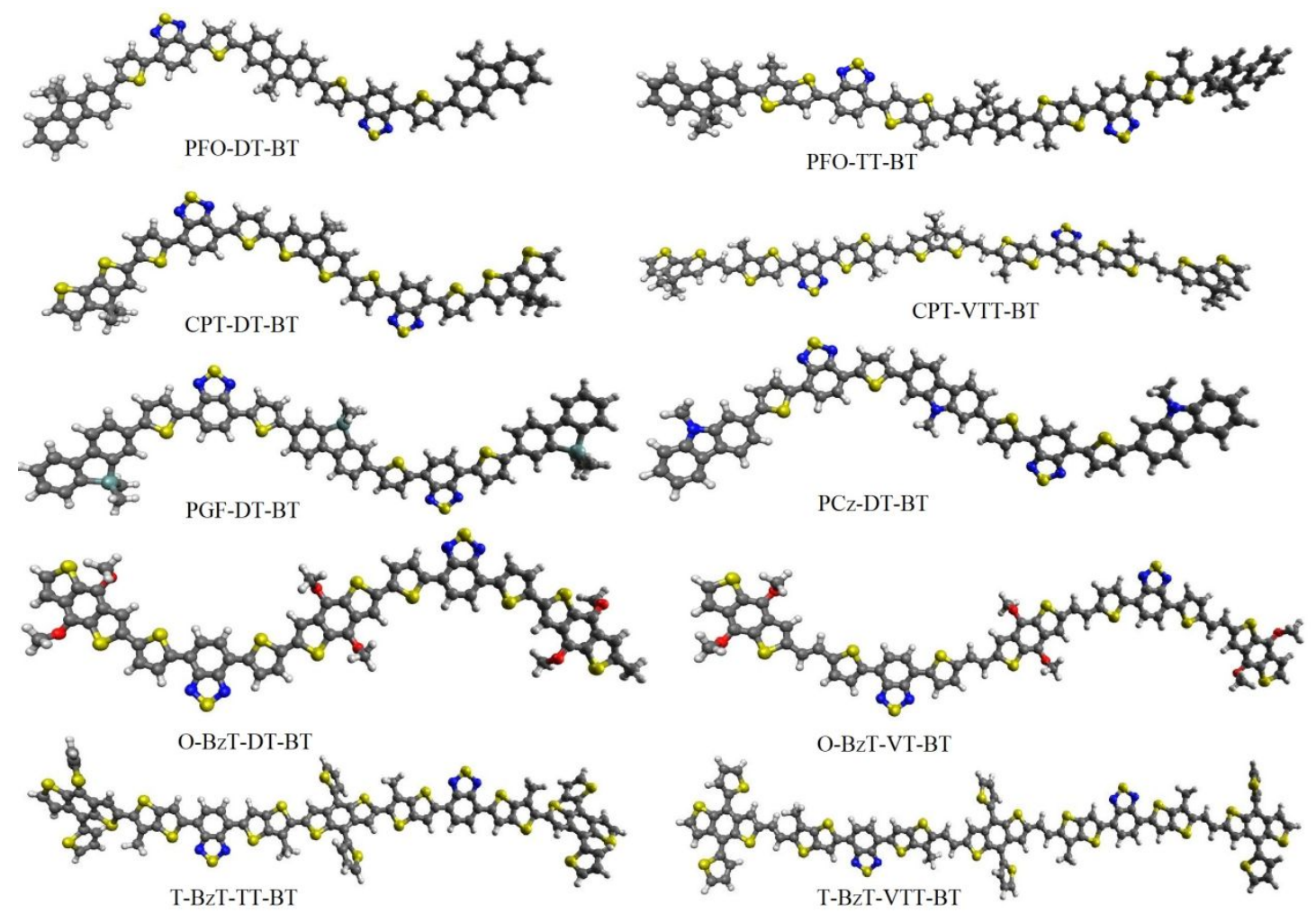

Figure S1- Selected optimized geometries for benzothiadiazole-based oligomers.

\section{2-Density of states: validation}

To assure the solid-sate calculations represent well the electronic structure for conjugated polymers in a molecular structure, we have validated the plane-wave augmented methodology against the gaussian basis sets by calculating the pDOS for PFO-DT-BT, our system of reference. In our previous work, ${ }^{1}$ the AOMIX code have been utilized to establish the moiety contributions for the total density of states. The main findings indicated the thiophene is the highest contributing group for the HOMO, whereas the benzothiadiazole unit compose the first unoccupied orbital. In Fig. S2, we observe that the VASP calculations provide a fair agreement with the published report, although the peaks are expected to present higher localization due to the molecular structure. The code is found especially important to unveil the atomic chemical environment and its influence 
to the distribution of energy states. For instance, the $\mathrm{Csp} p^{2}$ states from thiophene dominate the HOMO peak, meanwhile the LUMO is basically composed by $\mathrm{N} 2 p$ states from benzothiadiazole.

At this point, we emphasize that the energy values expressed by the pDOS plots in this work does not match the HOMO/LUMO values or the redox potentials obtained via reaction thermodynamics. Instead we have a qualitative information that can be used to provide a better understanding of the polymer electronic structures that are investigated in this project. For direct comparison to the energy levels, the band potentials should be calculated, which are out of scope of this work.

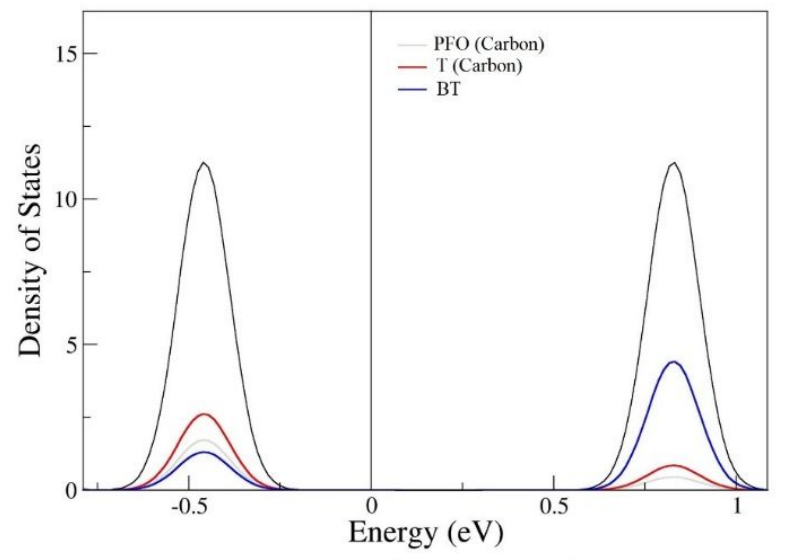

Figure S2- Projected DOS for PFO-DT-BT obtained at VASP for code validation. The fermi level is set at zero.

\section{3-Optical Properties}
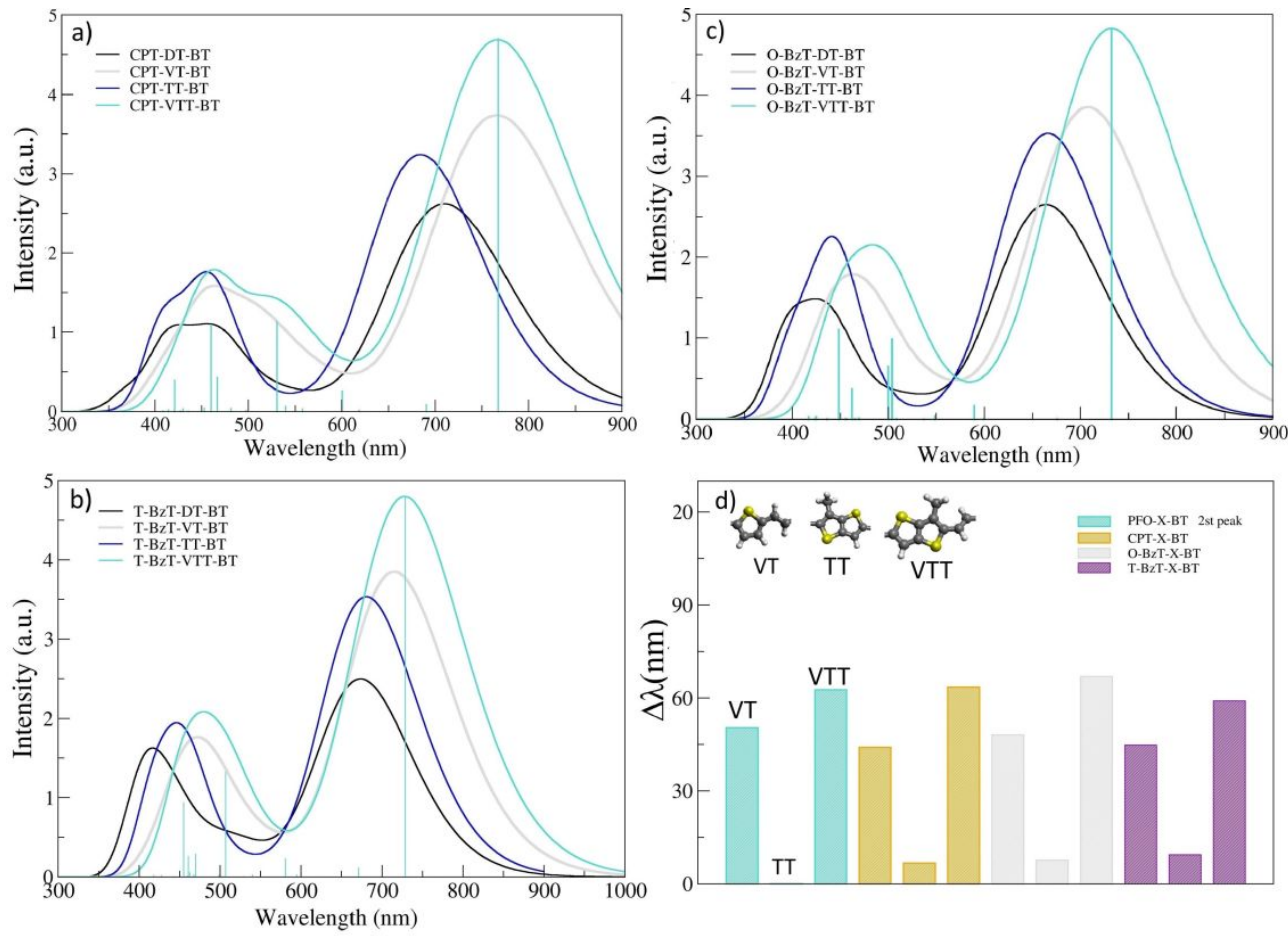

Figure S3- UV/Vis absorption spectra obtained at M06/6-311G(d,p) level of theory for cyclopentadithiophene (a), T-BzT (b) and O-BzT-based polymers (c). In (d) is shown the variation in $\lambda$ of the second main peak of absorption and the reference for each group. 


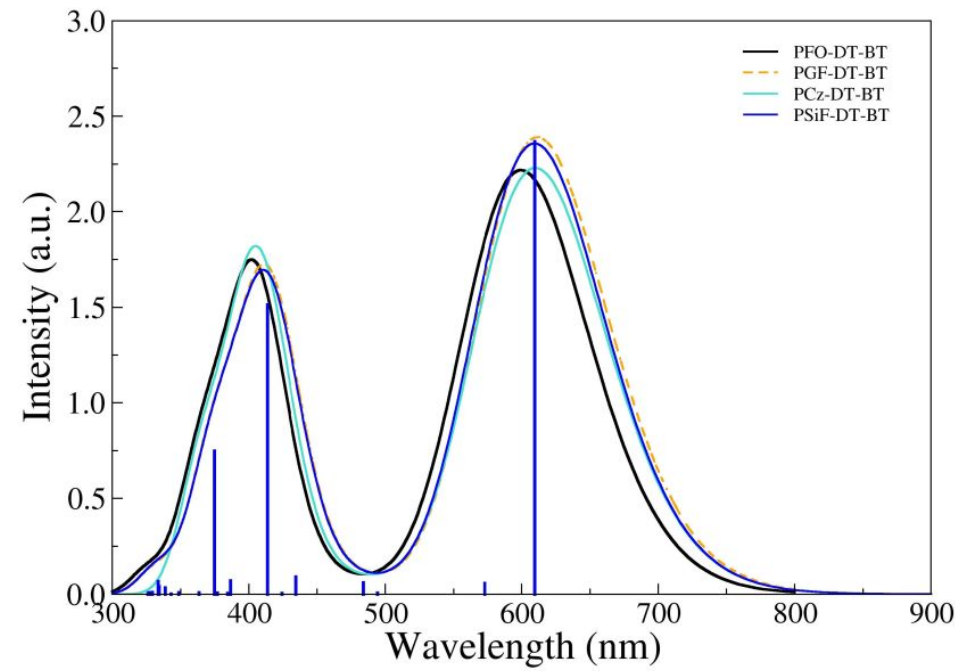

Figure S4- UV/Vis absorption spectra obtained at M06/6-311G(d,p) level of theory for fluorene-, germafluorene-, carbazole- and silafluorene-based polymers. The peak intensities are weighed by their oscillator strength.

Table S1- Optical Properties of the main singlet excited states obtained at the range 300-1000 nm within TDDFT methods. CT stands for an electronic transition with charge-transfer nature.

\begin{tabular}{|c|c|c|c|c|c|c|}
\hline \multirow{2}{*}{$\begin{array}{r}\text { System } \\
\text { PFO-DT-BT }\end{array}$} & \multirow{2}{*}{$\begin{array}{c}\lambda \\
(\mathbf{n m}) \\
\mathrm{S}_{1}: 600.58\end{array}$} & \multirow{2}{*}{$\begin{array}{c}\text { Character } \\
\text { CT }\end{array}$} & \multirow{2}{*}{$\begin{array}{c}\mathbf{f} \\
2.220\end{array}$} & \multicolumn{2}{|c|}{ Major contributions } & \multirow{2}{*}{$\begin{array}{c}\mathbf{E}_{\text {opt }} \\
(\mathbf{e V}) \\
2.06\end{array}$} \\
\hline & & & & $\mathrm{H} \rightarrow \mathrm{L}$ & $(76 \%)$ & \\
\hline & $\mathrm{S}_{7}: 404.76$ & $\pi \rightarrow \pi^{*}$ & 1.610 & $\mathrm{H} \rightarrow \mathrm{L}+2$ & $(80 \%)$ & $(1.90)^{2}$ \\
\hline \multirow[t]{3}{*}{ PFO-TT-BT } & $\mathrm{S}_{1}: 604.47$ & $\mathrm{CT}$ & 2.640 & $\mathrm{H} \rightarrow \mathrm{L}$ & $(67 \%)$ & 2.05 \\
\hline & $\mathrm{S}_{8}: 404.82$ & $\pi \rightarrow \pi^{*}$ & 1.466 & $\mathrm{H} \rightarrow \mathrm{L}+2$ & $(42 \%)$ & \\
\hline & $\mathrm{S}_{15}: 368.74$ & $\pi \rightarrow \pi^{*}$ & 0.582 & $\mathrm{H}>\mathrm{L}+4$ & $(31 \%)$ & \\
\hline \multirow[t]{3}{*}{ PFO-VT-BT } & $\mathrm{S}_{1}: 663.05$ & $\mathrm{CT}$ & 3.234 & $\mathrm{H} \rightarrow \mathrm{L}$ & $(75 \%)$ & 1.87 \\
\hline & $S_{7}: 455.12$ & $\pi \rightarrow \pi^{*}$ & 1.399 & $\mathrm{H} \rightarrow \mathrm{L}+2$ & $(78 \%)$ & \\
\hline & $\mathrm{S}_{11}: 413.15$ & $\pi \rightarrow \pi^{*}$ & 1.048 & $\mathrm{H} \rightarrow \mathrm{L}+4$ & $(25 \%)$ & \\
\hline \multirow[t]{3}{*}{ PFO-VTT-BT } & $\mathrm{S}_{1}: 680.37$ & $\mathrm{CT}$ & 4.032 & $\mathrm{H} \rightarrow \mathrm{L}$ & $(72 \%)$ & 1.82 \\
\hline & $S_{7}: 467.37$ & $\pi \rightarrow \pi^{*}$ & 2.220 & $\mathrm{H} \rightarrow \mathrm{L}+2$ & $(80 \%)$ & \\
\hline & $\mathrm{S}_{11}: 420.13$ & $\pi \rightarrow \pi^{*}$ & 1.240 & $\mathrm{H} \rightarrow \mathrm{L}+4$ & $(24 \%)$ & \\
\hline \multirow[t]{2}{*}{ PGF-DT-BT } & $\mathrm{S}_{1}: 611.69$ & $\mathrm{CT}$ & 2.391 & $\mathrm{H}>\mathrm{L}$ & $(76 \%)$ & 2.03 \\
\hline & $\mathrm{S}_{5}: 413.80$ & $\pi \rightarrow \pi^{*}$ & 1.548 & $\mathrm{H}>\mathrm{L}+2$ & $(81 \%)$ & $(1.67)^{2}$ \\
\hline \multirow[t]{2}{*}{ PCz-DT-BT } & S1: 611.00 & $\mathrm{CT}$ & 2.207 & $\mathrm{H}>\mathrm{L}$ & $(76 \%)$ & 2.03 \\
\hline & $\mathrm{S}_{4}: 407.37$ & $\pi \rightarrow \pi^{*}$ & 1.629 & $\mathrm{H}>\mathrm{L}+2$ & $(78 \%)$ & $(1.90)$ \\
\hline \multirow[t]{2}{*}{ PSiF-DT-BT } & $S_{1}: 609.61$ & $\mathrm{CT}$ & 2.367 & $\mathrm{H}>\mathrm{L}$ & $(76 \%)$ & 1.98 \\
\hline & $\mathrm{S}_{5}: 413.59$ & $\pi \rightarrow \pi^{*}$ & 1.513 & $\mathrm{H}>\mathrm{L}+2$ & $(81 \%)$ & $(1.85)^{2}$ \\
\hline \multirow[t]{2}{*}{ CPT-DT-BT } & $\mathrm{S}_{1}: 710.51$ & $\mathrm{CT}$ & 2.620 & $\mathrm{H} \rightarrow \mathrm{L}$ & $(87 \%)$ & 1.75 \\
\hline & $\mathrm{S}_{7}: 466.89$ & $\pi \rightarrow \pi^{*}$ & 0.930 & $\mathrm{H} \rightarrow \mathrm{L}+2$ & $(87 \%)$ & \\
\hline
\end{tabular}




\begin{tabular}{|c|c|c|c|c|c|c|}
\hline & $S_{11}: 413.71$ & $\pi \rightarrow \pi^{*}$ & 0.712 & $\mathrm{H} \rightarrow \mathrm{L}+4$ & $(46 \%)$ & \\
\hline \multirow[t]{3}{*}{ CPT-TT-BT } & $\mathrm{S}_{1}: 684.50$ & CT & 3.276 & $\mathrm{H} \rightarrow \mathrm{L}$ & $(80 \%)$ & 1.81 \\
\hline & $\mathrm{S}_{7}: 460.20$ & $\pi \rightarrow \pi^{*}$ & 1.633 & $\mathrm{H} \rightarrow \mathrm{L}+2$ & $(84 \%)$ & \\
\hline & $\mathrm{S}_{11}: 408.63$ & $\pi \rightarrow \pi^{*}$ & 0.634 & $\mathrm{H} \rightarrow \mathrm{L}+4$ & $(23 \%)$ & \\
\hline \multirow[t]{3}{*}{ CPT-VT-BT } & $\mathrm{S}_{1}: 766.28$ & $\mathrm{CT}$ & 3.747 & $\mathrm{H} \rightarrow \mathrm{L}$ & $(84 \%)$ & 1.62 \\
\hline & $\mathrm{S}_{7}: 510.91$ & $\pi \rightarrow \pi^{*}$ & 1.000 & $\mathrm{H} \rightarrow \mathrm{L}+2$ & $(83 \%)$ & \\
\hline & $S_{10}: 455.70$ & $\pi \rightarrow \pi^{*}$ & 1.040 & $\mathrm{H} \rightarrow \mathrm{L}+4$ & $(43 \%)$ & \\
\hline \multirow[t]{3}{*}{ CPT-VTT-BT } & $\mathrm{S}_{1}: 767.41$ & $\mathrm{CT}$ & 4.700 & $\mathrm{H} \rightarrow \mathrm{L}$ & $(81 \%)$ & 1.62 \\
\hline & $\mathrm{S}_{7}: 530.39$ & $\pi \rightarrow \pi^{*}$ & 1.135 & $\mathrm{H} \rightarrow \mathrm{L}+2$ & $(85 \%)$ & \\
\hline & $\mathrm{S}_{11}: 459.84$ & $\pi \rightarrow \pi^{*}$ & 1.090 & $\mathrm{H} \rightarrow \mathrm{L}+3$ & $(37 \%)$ & \\
\hline \multirow[t]{3}{*}{ O-BzT-DT-BT } & $\mathrm{S}_{1}: 663.62$ & CT & 2.647 & $\mathrm{H} \rightarrow \mathrm{L}$ & $(81 \%)$ & 1.87 \\
\hline & $\mathrm{S}_{7}: 436.75$ & $\pi \rightarrow \pi^{*}$ & 0.840 & $\mathrm{H} \rightarrow \mathrm{L}+2$ & $(65 \%)$ & \\
\hline & $\mathrm{S}_{11}: 395.31$ & $\pi \rightarrow \pi^{*}$ & 1.050 & $\mathrm{H} \rightarrow \mathrm{L}+4$ & $(34 \%)$ & \\
\hline \multirow[t]{2}{*}{ O-BzT-TT-BT } & $\mathrm{S}_{1}: 665.67$ & CT & 3.523 & $\mathrm{H} \rightarrow \mathrm{L}$ & $(75 \%)$ & 1.86 \\
\hline & $\mathrm{S}_{9}: 444.34$ & $\pi \rightarrow \pi^{*}$ & 1.966 & $\mathrm{H} \rightarrow \mathrm{L}+2$ & $(79 \%)$ & \\
\hline \multirow[t]{2}{*}{ O-BzT-VT-BT } & $\mathrm{S}_{1}: 707.61$ & $\mathrm{CT}$ & 3.840 & $\mathrm{H} \rightarrow \mathrm{L}$ & $(80 \%)$ & 1.75 \\
\hline & $\mathrm{S}_{8}: 484.75$ & $\pi \rightarrow \pi^{*}$ & 1.113 & $\mathrm{H} \rightarrow \mathrm{L}+2$ & $(66 \%)$ & \\
\hline \multirow[t]{4}{*}{ O-BzT-VTT-BT } & $\mathrm{S}_{1}: 732.03$ & CT & 4.816 & $\mathrm{H} \rightarrow \mathrm{L}$ & $(76 \%)$ & 1.69 \\
\hline & $S \cdot 50361$ & $\pi \rightarrow \pi *$ & 0985 & $\mathrm{H} \rightarrow \mathrm{L}+2$ & $(29 \%)$ & \\
\hline & & & & $\mathrm{H}-3 \rightarrow \mathrm{L}+1$ & $(30 \%)$ & \\
\hline & $\mathrm{S}_{15}: 448.15$ & $\pi \rightarrow \pi^{*}$ & 1.106 & $\mathrm{H} \rightarrow \mathrm{L}+4$ & $(29 \%)$ & \\
\hline \multirow[t]{3}{*}{ T-BzT-DT-BT } & $S_{1}: 673.60$ & $\mathrm{CT}$ & 2.495 & $\mathrm{H} \rightarrow \mathrm{L}$ & $(80 \%)$ & 1.84 \\
\hline & $\mathrm{S}_{11}: 447.85$ & $\pi \rightarrow \pi^{*}$ & 0.735 & $\mathrm{H} \rightarrow \mathrm{L}+2$ & $(80 \%)$ & \\
\hline & $\mathrm{S}_{15}: 412.00$ & $\pi \rightarrow \pi^{*}$ & 1.116 & $\mathrm{H} \rightarrow \mathrm{L}+4$ & $(30 \%)$ & \\
\hline \multirow[t]{3}{*}{ T-BzT-TT-BT } & $\mathrm{S}_{1}: 680.59$ & CT & 3.555 & $\mathrm{H} \rightarrow \mathrm{L}$ & $(77 \%)$ & 1.82 \\
\hline & $\mathrm{S}_{9}: 457.18$ & $\pi \rightarrow \pi^{*}$ & 1.424 & $\mathrm{H} \rightarrow \mathrm{L}+2$ & $(82 \%)$ & \\
\hline & $\mathrm{S}_{15}: 418.04$ & $\pi \rightarrow \pi^{*}$ & 1.064 & $\mathrm{H} \rightarrow \mathrm{L}+4$ & $(25 \%)$ & \\
\hline \multirow[t]{2}{*}{ T-BzT-VT-BT } & $\mathrm{S}_{1}: 714.68$ & $\mathrm{CT}$ & 3.846 & $\mathrm{H} \rightarrow \mathrm{L}$ & $(80 \%)$ & 1.73 \\
\hline & $S_{9}: 492.62$ & $\pi \rightarrow \pi^{*}$ & 0.557 & $\mathrm{H} \rightarrow \mathrm{L}+2$ & $(76 \%)$ & \\
\hline \multirow[t]{3}{*}{ T-BzT-VTT-BT } & $\mathrm{S}_{1}: 728.71$ & $\mathrm{CT}$ & 4.794 & $\mathrm{H} \rightarrow \mathrm{L}$ & $(77 \%)$ & 1.70 \\
\hline & $\mathrm{S}_{9}: 506.88$ & $\pi \rightarrow \pi^{*}$ & 1.325 & $\mathrm{H} \rightarrow \mathrm{L}+2$ & $(80 \%)$ & \\
\hline & $\mathrm{S}_{7}: 455.07$ & $\pi \rightarrow \pi^{*}$ & 0.937 & $\mathrm{H} \rightarrow \mathrm{L}+4$ & $(24 \%)$ & \\
\hline
\end{tabular}



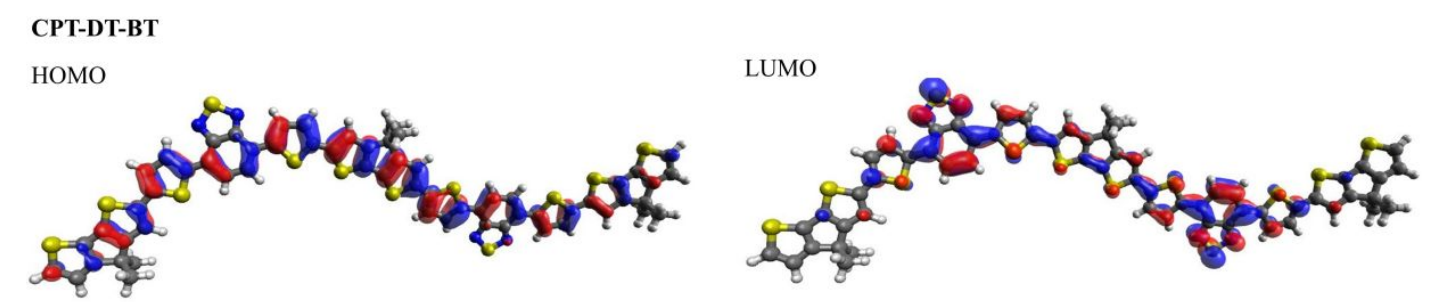

LUMO+2

LUMO+4
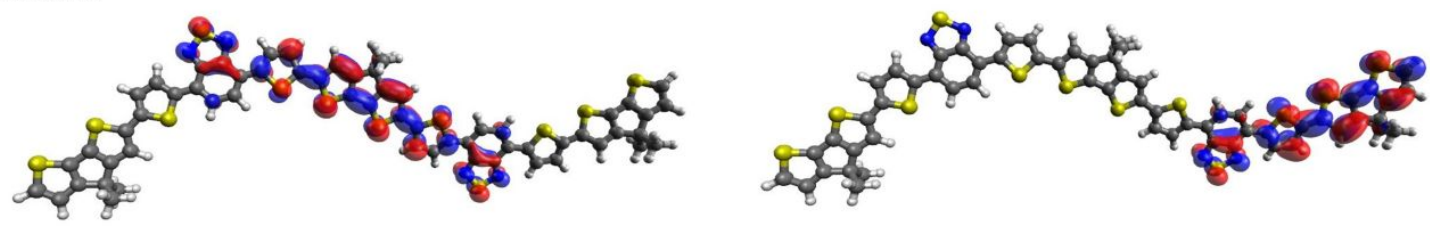

Figure S5- $\mathrm{HOMO} / \mathrm{LUMO}+n$ spatial distribution in the cyclopentadithiophene-based dimer (CPT-DT-BT) at isovalue 0.02. Yellow, blue, white and grey spheres represent sulfur, nitrogen, hydrogen and carbon atoms, respectively. 

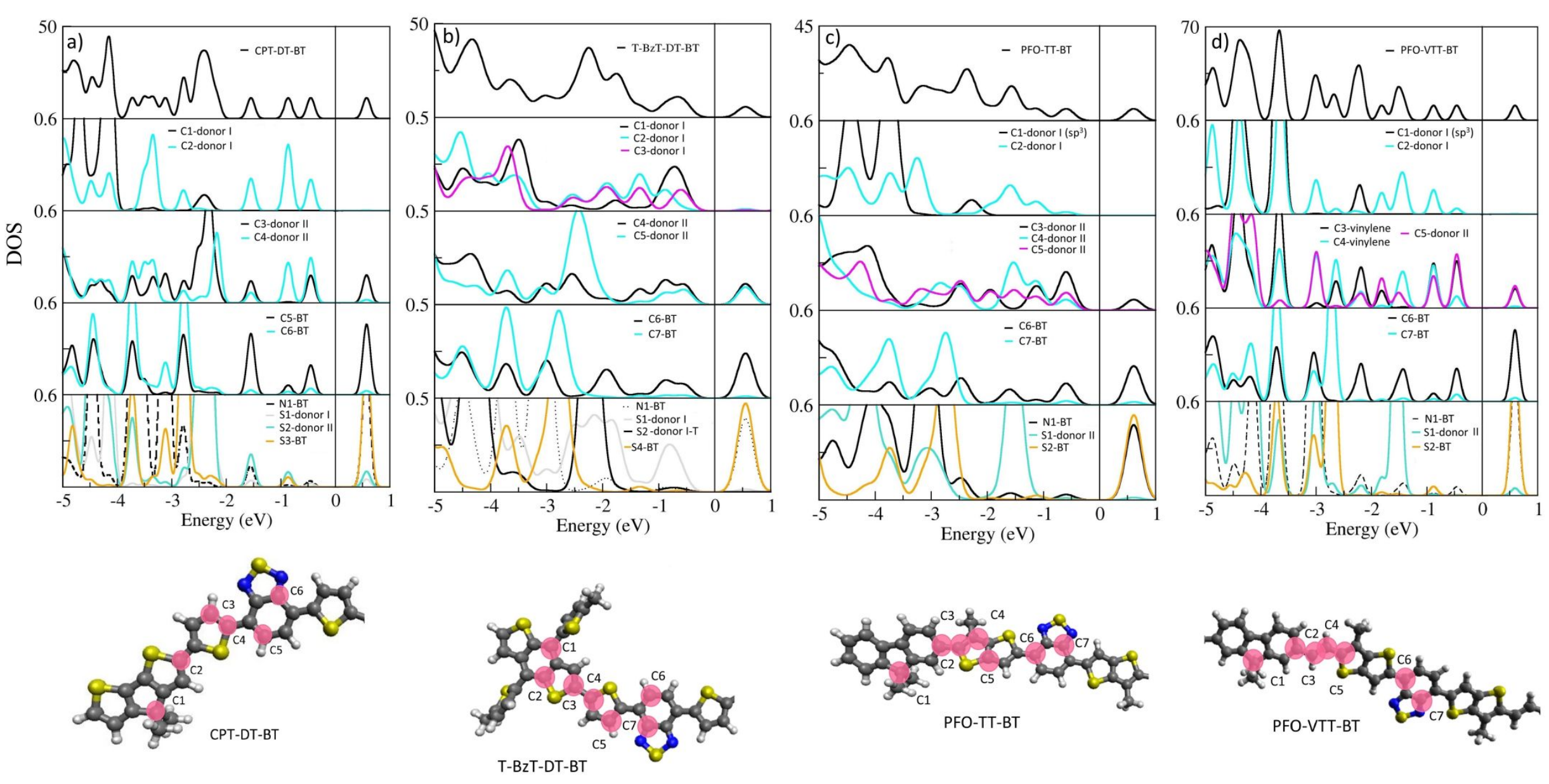

Figure S6- Density of states for selected systems. Code: VASP, $400 \mathrm{eV}$.

S6 
Table S4- Ground state potentials in absolute values as obtained via DFT approach. Level of theory: M06/6-31G(d) for optimizations and frequencies, 6-311G(d,p) for single point calculations in gas and solvated phase. Experimental values are shown in parenthesis, being $\left(^{*}\right)$ applied to the values that were originally obtained via optical gap. Solvent: water.

\begin{tabular}{|c|c|c|c|c|}
\hline Material & $\begin{array}{l}\phi_{P^{+}} / P \\
\text { (V) }\end{array}$ & $\begin{array}{l}\phi_{P^{-} / P} \\
(\mathbf{V})\end{array}$ & $\begin{array}{l}E_{g} \\
(e V)\end{array}$ & Ref. \\
\hline PFO-DT-BT & $\begin{array}{l}5.33 \\
(5.34)\end{array}$ & $\begin{array}{l}3.14 \\
(3.44)\end{array}$ & $\begin{array}{l}2.19 \\
(1.90)\end{array}$ & $\begin{array}{l}\text { Alghamdi et } \\
\text { al. }{ }^{3}\end{array}$ \\
\hline $\begin{array}{l}\text { PFO-TT-BT } \\
\text { (PF-tt-BT) }\end{array}$ & $\begin{array}{l}5.15 \\
(5.43)\end{array}$ & $\begin{array}{l}3.28 \\
(3.66)\end{array}$ & $\begin{array}{l}1.87 \\
(1.77)\end{array}$ & Li et al. ${ }^{4}$ \\
\hline PFO-VT-BT & 5.15 & 3.26 & 1.89 & \\
\hline PFO-VTT-BT & 4.98 & 3.30 & 1.68 & \\
\hline PGF-DT-BT & $\begin{array}{l}5.10 \\
(5.58)\end{array}$ & $\begin{array}{l}3.35 \\
(3.91)\end{array}$ & $\begin{array}{l}1.75 \\
(1.67)\end{array}$ & Allard et al. ${ }^{2}$ \\
\hline $\begin{array}{l}\text { PCz-DT-BT } \\
\text { (PCz3TB) }\end{array}$ & $\begin{array}{l}5.19 \\
(5.24) \\
(5.35)\end{array}$ & $\begin{array}{l}3.31 \\
(3.34)^{*} \\
(3.42)\end{array}$ & $\begin{array}{l}1.88 \\
(1.90)^{* 5} \\
(1.93)^{3}\end{array}$ & $\begin{array}{l}\text { Lim et al. }{ }^{5} \\
\text { Alghamdi et } \\
\text { al. }{ }^{3}\end{array}$ \\
\hline $\begin{array}{l}\text { PSiF-DT-BT }^{6} \text { : } \\
\text { (PSiF-DBT) }\end{array}$ & $\begin{array}{l}5.22 \\
(5.39)\end{array}$ & $\begin{array}{l}3.24 \\
(3.57)^{*}\end{array}$ & $\begin{array}{l}1.98 \\
(1.82)^{*}\end{array}$ & Wang et al. ${ }^{6}$ \\
\hline $\begin{array}{l}\text { CPT-DT-BT } \\
\text { CPT-TT-BT } \\
\text { CPT-VT-BT } \\
\text { CPT-VTT-BT }\end{array}$ & $\begin{array}{l}4.83 \\
4.93 \\
4.75 \\
4.66\end{array}$ & $\begin{array}{l}3.27 \\
3.17 \\
3.25 \\
3.24\end{array}$ & $\begin{array}{l}1.56 \\
1.76 \\
1.50 \\
1.42\end{array}$ & \\
\hline $\begin{array}{l}\text { O-BzT-DT-BT } \\
\text { O-BzT-TT-BT } \\
\text { (PBDT-tt-BT) } \\
\text { O-BzT-VT-BT } \\
\text { O-BzT-VTT-BT }\end{array}$ & $\begin{array}{l}5.28 \\
5.11 \\
(5.21) \\
5.15 \\
4.97\end{array}$ & $\begin{array}{l}3.24 \\
3.33 \\
(3.78) \\
3.36 \\
3.40\end{array}$ & $\begin{array}{l}2.04 \\
1.78 \\
(1.46) \\
1.79 \\
1.57\end{array}$ & Li et al. ${ }^{4}$ \\
\hline $\begin{array}{l}\text { T-BzT-DT-BT } \\
\text { T-BzT-TT-BT } \\
\text { T-BzT-VT-BT } \\
\text { T-BzT-VTT-BT }\end{array}$ & $\begin{array}{l}5.19 \\
5.10 \\
5.10 \\
4.97\end{array}$ & $\begin{array}{l}3.34 \\
3.34 \\
3.28 \\
3.33\end{array}$ & $\begin{array}{l}1.85 \\
1.76 \\
1.82 \\
1.64\end{array}$ & \\
\hline
\end{tabular}

Table S5- Ground state potentials in absolute values as obtained via DFT approach. Level of theory: M06/6-31G(d) for optimizations and frequencies, 6-311G(d,p) for single point calculations in gas and solvated phase. Experimental values are shown in parenthesis, being $\left(^{*}\right)$ applied to the values that were originally obtained via optical gap. Solvent: 1-bromooctane.

Material $\quad \begin{array}{llll}\phi_{P^{+} / P} & \phi_{P^{-} / P} & \mathbf{E}_{\mathbf{g}} & \text { Ref. }\end{array}$




\begin{tabular}{|c|c|c|c|c|}
\hline & (V) & $(\mathrm{V})$ & $(\mathrm{eV})$ & \\
\hline PFO-DT-BT & $\begin{array}{l}5.44 \\
(5.34)\end{array}$ & $\begin{array}{l}2.87 \\
(3.44)\end{array}$ & $\begin{array}{l}2.57 \\
(1.90)\end{array}$ & $\begin{array}{l}\text { Alghamdi et } \\
\text { al. }^{3}\end{array}$ \\
\hline $\begin{array}{l}\text { PFO-TT-BT } \\
\text { (PF-tt-BT) }\end{array}$ & $\begin{array}{l}5.28 \\
(5.43)\end{array}$ & $\begin{array}{l}2.99 \\
(3.66)\end{array}$ & $\begin{array}{l}2.29 \\
(1.77)\end{array}$ & Li et al. ${ }^{4}$ \\
\hline PFO-VT-BT & 5.24 & 3.00 & 2.24 & \\
\hline PFO-VTT-BT & 5.08 & 3.03 & 2.05 & \\
\hline PGF-DT-BT & $\begin{array}{l}5.21 \\
(5.58)\end{array}$ & $\begin{array}{l}3.08 \\
(3.91)\end{array}$ & $\begin{array}{l}2.13 \\
(1.67)\end{array}$ & Allard et al. ${ }^{2}$ \\
\hline \multirow{2}{*}{$\begin{array}{l}\text { PCz-DT-BT } \\
\text { (PCz3TB) }\end{array}$} & 5.29 & 3.04 & 2.26 & \\
\hline & $\begin{array}{l}(5.24) \\
(5.35)\end{array}$ & $\begin{array}{l}(3.34)^{*} \\
(3.42)\end{array}$ & $\begin{array}{l}(1.90)^{* 5} \\
(1.93)^{3}\end{array}$ & $\begin{array}{l}\text { Lim et al. }{ }^{5} \\
\text { Alghamdi et } \\
\text { al. }{ }^{3}\end{array}$ \\
\hline $\begin{array}{l}\text { PSiF-DT-BT }^{6} \text { : } \\
(\text { PSiF-DBT) }\end{array}$ & $\begin{array}{l}5.33 \\
(5.39)\end{array}$ & $\begin{array}{l}2.97 \\
(3.57)^{*}\end{array}$ & $\begin{array}{l}2.36 \\
(1.82)^{*}\end{array}$ & Wang et al. ${ }^{6}$ \\
\hline $\begin{array}{l}\text { CPT-DT-BT } \\
\text { CPT-TT-BT } \\
\text { CPT-VT-BT } \\
\text { CPT-VTT-BT }\end{array}$ & $\begin{array}{l}4.96 \\
5.06 \\
4.88 \\
4.78\end{array}$ & $\begin{array}{l}3.04 \\
2.91 \\
3.07 \\
3.06\end{array}$ & $\begin{array}{l}1.92 \\
2.14 \\
1.82 \\
1.73\end{array}$ & \\
\hline $\begin{array}{l}\text { O-BzT-DT-BT } \\
\text { O-BzT-TT-BT } \\
\text { (PBDT-tt-BT) } \\
\text { O-BzT-VT-BT } \\
\text { O-BzT-VTT-BT }\end{array}$ & $\begin{array}{l}5.40 \\
5.22 \\
(5.21) \\
5.27 \\
5.08\end{array}$ & $\begin{array}{l}2.98 \\
3.09 \\
(3.78) \\
3.15 \\
3.16\end{array}$ & $\begin{array}{l}2.42 \\
2.14 \\
(1.46) \\
2.12 \\
1.92\end{array}$ & Li et al. ${ }^{4}$ \\
\hline $\begin{array}{l}\text { T-BzT-DT-BT } \\
\text { T-BzT-TT-BT } \\
\text { T-BzT-VT-BT } \\
\text { T-BzT-VTT-BT }\end{array}$ & $\begin{array}{l}5.28 \\
5.21 \\
5.21 \\
5.07\end{array}$ & $\begin{array}{l}3.14 \\
3.09 \\
3.11 \\
3.09\end{array}$ & $\begin{array}{l}2.14 \\
2.12 \\
2.10 \\
1.98\end{array}$ & \\
\hline
\end{tabular}

Table S6- Fundamental and optical gap, as well as exciton binding energies $\left(\mathrm{E}_{\mathrm{b}}\right)$ obtained in the ground-state geometries. Level of theory: M06/6-311G(d,p). In the last columns, $\mathrm{E}_{\mathrm{b}}$ are computed in water or gas phase.

\begin{tabular}{|c|c|c|c|c|c|c|}
\hline \multirow[t]{2}{*}{ Ref. } & \multirow[t]{2}{*}{ System } & \multicolumn{2}{|c|}{$\mathrm{E}_{\mathrm{f}}(\mathrm{IP}-\mathrm{EA})$} & \multirow{2}{*}{$\begin{array}{l}\mathrm{E}_{\text {opt }} \text { (gas } \\
\text { phase) }\end{array}$} & \multicolumn{2}{|c|}{$\mathrm{E}_{\mathrm{b}}$} \\
\hline & & $\begin{array}{l}\text { +solv } \\
\text { (water) }\end{array}$ & - no solv & & $\begin{array}{l}+ \text { solv } \\
\text { (water) }\end{array}$ & -no solv \\
\hline 1 & PFO-DT-BT & 2.248 & 3.870 & 2.027 & 0.221 & 1.844 \\
\hline 2 & PFO-TT-BT & 2.088 & 3.717 & 2.051 & 0.038 & 1.667 \\
\hline 3 & PFO-VT-BT & 2.022 & 3.502 & 1.870 & 0.152 & 1.632 \\
\hline 4 & PFO-VTT-BT & 1.865 & 3.333 & 1.822 & 0.042 & 1.511 \\
\hline 5 & PGF-DT-BT & 2.255 & 3.858 & 2.027 & 0.222 & 1.831 \\
\hline 6 & PCz-DT-BT & 2.249 & 3.880 & 2.029 & 0.225 & 1.850 \\
\hline
\end{tabular}




\begin{tabular}{lllllll}
\hline 7 & PSiF-DT-BT & 2.258 & 3.869 & 2.034 & $\mathbf{0 . 2 2 5}$ & $\mathbf{1 . 8 3 6}$ \\
8 & CPT-DT-BT & 1.776 & 3.431 & 1.745 & $\mathbf{0 . 0 3}$ & $\mathbf{1 . 6 8 6}$ \\
9 & CPT-TT-BT & 1.800 & 3.321 & 1.811 & $\mathbf{- 0 . 0 1}$ & $\mathbf{1 . 5 0 9}$ \\
10 & CPT-VT-BT & 1.622 & 3.165 & 1.618 & $\mathbf{0 . 0 0 4}$ & $\mathbf{1 . 5 4 7}$ \\
11 & CPT-VTT-BT & 1.526 & 3.044 & 1.616 & $\mathbf{- 0 . 0 9}$ & $\mathbf{1 . 4 2 8}$ \\
& & & & & & \\
12 & O-BzT-DT-BT & 2.060 & 3.605 & 1.868 & $\mathbf{0 . 1 9 2}$ & $\mathbf{1 . 7 3 7}$ \\
13 & O-BzT-TT-BT & 1.980 & 3.474 & 1.863 & $\mathbf{0 . 1 1 8}$ & $\mathbf{1 . 6 1 1}$ \\
14 & O-BzT-VT-BT & 1.912 & 3.351 & 1.752 & $\mathbf{0 . 1 5 9}$ & $\mathbf{1 . 5 9 9}$ \\
15 & O-BzT-VTT-BT & 1.779 & 3.168 & 1.694 & $\mathbf{0 . 0 8 5}$ & $\mathbf{1 . 4 7 4}$ \\
& & & & & & \\
16 & T-BzT-DT-BT & 2.024 & 3.509 & 1.841 & $\mathbf{0 . 1 8 3}$ & $\mathbf{1 . 6 6 8}$ \\
17 & T-BzT-TT-BT & 1.931 & 3.394 & 1.822 & $\mathbf{0 . 1 0 9}$ & $\mathbf{1 . 5 7 2}$ \\
18 & T-BzT-VT-BT & 1.895 & 3.294 & 1.735 & $\mathbf{0 . 1 6 0}$ & $\mathbf{1 . 5 5 9}$ \\
19 & T-BzT-VTT-BT & & 3.169 & 1.701 & $\mathbf{0 . 0 8 0}$ & $\mathbf{1 . 4 6 7}$ \\
\hline
\end{tabular}

Table S7- Fundamental and optical gap, as well as exciton binding energies $\left(E_{b}\right)$ obtained in the ground-state geometries. Level of theory: M06/6-311G(d,p) in 1 -bromooctane- $(\varepsilon=5.04)$.

\begin{tabular}{lllll}
\hline Ref. & System & $\begin{array}{l}E_{\mathrm{f}}(\mathrm{IP}- \\
\text { EA)solv }\end{array}$ & $\begin{array}{l}\mathrm{E}_{\text {opt }} \text { (gas } \\
\text { phase })\end{array}$ & $\begin{array}{l}\mathrm{E}_{\mathrm{b}} \\
+ \text { solv }\end{array}$ \\
1 & PFO-DT-BT & 2.559 & 2.027 & $\mathbf{0 . 5 3 2}$ \\
2 & PFO-TT-BT & 2.516 & 2.051 & $\mathbf{0 . 4 6 5}$ \\
3 & PFO-VT-BT & 2.309 & 1.870 & $\mathbf{0 . 4 3 4}$ \\
4 & PFO-VTT-BT & 2.220 & 1.822 & $\mathbf{0 . 3 9 8}$ \\
& & & & \\
5 & PGF-DT-BT & 2.558 & 2.027 & $\mathbf{0 . 5 3 2}$ \\
6 & PCz-DT-BT & 2.566 & 2.029 & $\mathbf{0 . 5 3 7}$ \\
7 & PSiF-DT-BT & 2.568 & 2.034 & $\mathbf{0 . 5 3 4}$ \\
& & & & \\
8 & CPT-DT-BT & 2.108 & 1.745 & $\mathbf{0 . 3 6 3}$ \\
9 & CPT-TT-BT & 2.115 & 1.811 & $\mathbf{0 . 3 0 3}$ \\
10 & CPT-VT-BT & 1.943 & 1.618 & $\mathbf{0 . 3 2 5}$ \\
11 & CPT-VTT-BT & 1.901 & 1.616 & $\mathbf{0 . 2 9 3}$ \\
& & & & \\
12 & O-BzT-DT-BT & 2.313 & 1.868 & $\mathbf{0 . 4 9 3}$ \\
13 & O-BzT-TT-BT & 2.344 & 1.863 & $\mathbf{0 . 4 8 1}$ \\
14 & O-BzT-VT-BT & 2.199 & 1.752 & $\mathbf{0 . 4 4 7}$ \\
15 & O-BzT-VTT-BT & 2.126 & 1.694 & $\mathbf{0 . 4 3 3}$ \\
& & & & \\
16 & T-BzT-DT-BT & 2.322 & 1.841 & $\mathbf{0 . 4 8 1}$ \\
17 & T-BzT-TT-BT & 2.285 & 1.822 & $\mathbf{0 . 4 6 3}$ \\
18 & T-BzT-VT-BT & 2.176 & 1.735 & $\mathbf{0 . 4 4 2}$ \\
19 & T-BzT-VTT-BT & 2.119 & 1.701 & $\mathbf{0 . 4 1 7}$ \\
\hline
\end{tabular}

Table S8- Fundamental and optical gap, as well as exciton binding energies $\left(E_{b}\right)$ obtained in the ground-state geometries. Level of theory: M06/6-311G $(\mathrm{d}, \mathrm{p})$ in acetonitrile:35.65.

\begin{tabular}{lllll}
\hline Ref. & System & $\begin{array}{l}\mathrm{E}_{\mathrm{f}}(\mathrm{IP}- \\
\text { EA)solv }\end{array}$ & $\begin{array}{l}\mathrm{E}_{\text {opt }} \text { (gas } \\
\text { phase })\end{array}$ & $\mathrm{E}_{\mathrm{b}}+$ solv \\
\hline
\end{tabular}




\begin{tabular}{lllll}
\hline 1 & PFO-DT-BT & 2.274 & 2.027 & $\mathbf{0 . 2 4 8}$ \\
2 & PFO-TT-BT & 2.128 & 2.051 & $\mathbf{0 . 0 7 6}$ \\
3 & PFO-VT-BT & 2.047 & 1.870 & $\mathbf{0 . 1 7 7}$ \\
4 & PFO-VTT-BT & 1.899 & 1.822 & $\mathbf{0 . 0 7 8}$ \\
& & & & \\
5 & PGF-DT-BT & 2.275 & 2.027 & $\mathbf{0 . 2 4 9}$ \\
6 & PCz-DT-BT & 2.281 & 2.029 & $\mathbf{0 . 2 5 2}$ \\
7 & PSiF-DT-BT & 2.285 & 2.034 & $\mathbf{0 . 2 5 1}$ \\
& & & & \\
8 & CPT-DT-BT & 1.805 & 1.745 & $\mathbf{0 . 0 6 0}$ \\
9 & CPT-TT-BT & 1.828 & 1.811 & $\mathbf{0 . 0 1 7}$ \\
10 & CPT-VT-BT & 1.606 & 1.618 & $\mathbf{- 0 . 0 1 2}$ \\
11 & CPT-VTT-BT & 1.561 & 1.616 & $\mathbf{- 0 . 0 5 4}$ \\
& & & & \\
12 & O-BzT-DT-BT & 2.086 & 1.868 & $\mathbf{0 . 2 1 8}$ \\
13 & O-BzT-TT-BT & 2.014 & 1.863 & $\mathbf{0 . 1 5 1}$ \\
14 & O-BzT-VT-BT & 1.937 & 1.752 & $\mathbf{0 . 1 8 5}$ \\
15 & O-BzT-VTT-BT & 1.810 & 1.694 & $\mathbf{0 . 1 1 6}$ \\
& & & & \\
16 & T-BzT-DT-BT & 2.050 & 1.841 & $\mathbf{0 . 2 1 0}$ \\
17 & T-BzT-TT-BT & 1.963 & 1.822 & $\mathbf{0 . 1 4 2}$ \\
18 & T-BzT-VT-BT & 1.920 & 1.735 & $\mathbf{0 . 1 8 5}$ \\
19 & T-BzT-VTT-BT & 1.809 & 1.701 & $\mathbf{0 . 1 0 7}$ \\
\hline
\end{tabular}

Table S8- Donor/Acceptor ratio in the HOMO and LUMO composition. The orbitals composition was obtained by projecting the molecular orbitals into orbital belong to two different fragments: the Donor I and Donor II combination forming one of the fragments and the acceptor unit (BT for all systems) the other fragment. Level of theory: M06/6$311 \mathrm{G}(\mathrm{d}, \mathrm{p})$.

\begin{tabular}{ccc}
\hline System & D/A ratio & D/A ratio \\
PFO-DT-BT & 3.68 & 0.32 \\
PFO-VTT-BT & 6.61 & 0.31 \\
& & \\
CPT-DT-BT & 6.24 & 0.43 \\
CPT-VTT-BT & 10.90 & 0.43 \\
& & \\
O-BzT-DT-BT & 5.32 & 0.44 \\
O-BzT-VTT-BT & 9.30 & 0.42 \\
& & \\
T-BzT-DT-BT & 6.19 & 0.35 \\
T-BzT-VTT-BT & 9.07 & 0.41 \\
\hline
\end{tabular}

\section{4-References}

(1) Damas, G.; Marchiori, C. F. N.; Araujo, C. M. On the Design of Donor-Acceptor Conjugated Polymers for Photocatalytic Hydrogen Evolution Reaction: FirstPrinciples Theory-Based Assessment. Journal of Physical Chemistry C. 2018.

(2) Allard, N.; Aïch, R. B.; Gendron, D.; Boudreault, P. L. T.; Tessier, C.; Alem, S.; Tse, S. C.; Tao, Y.; Leclerc, M. Germafluorenes: New Heterocycles for Plastic Electronics. Macromolecules 2010, 43 (5), 2328-2333. 
(3) Alghamdi, A. A. B.; Watters, D. C.; Yi, H.; Al-Faifi, S.; Almeataq, M. S.; Coles, D.; Kingsley, J.; Lidzey, D. G.; Iraqi, A. Selenophene vs. Thiophene in Benzothiadiazole-Based Low Energy Gap Donor-Acceptor Polymers for Photovoltaic Applications. J. Mater. Chem. A 2013, 1 (16), 5165-5171.

(4) Li, Z.; Huo, L.; Guo, X.; Yong, W.; Zhang, S.; Fan, H. Synthesis and Photovoltaic Properties of D- $\pi$-A Copolymers Based on thieno[3,2-B]thiophene Bridge Unit. Polym. (United Kingdom) 2013, 54 (22), 6150-6157.

(5) Lim, E.; Lee, K. K.; Lee, S. Synthesis and Characterization of CarbazoleThiophene-Based Conjugated Polymers for Organic Photovoltaic Cells. Mol. Cryst. Liq. Cryst. 2011, 551, 130-137.

(6) Wang, E.; Wang, L.; Lan, L.; Luo, C.; Zhuang, W.; Wang, E.; Wang, L.; Lan, L.; Luo, C.; Zhuang, W. High-Performance Polymer Heterojunction Solar Cells of a Polysilafluorene Derivative High-Performance Polymer Heterojunction Solar Cells of a Polysilafluorene Derivative. Cit. Appl. Phys. Lett. Phys. Lett. Appl. Phys. Lett 2012, 33307 (2008), 1-4. 